\title{
INSTABILITY OF g-MODE OSCILLATIONS \\ IN WHITE DWARF STARS
}

\author{
Douglas A. Keeley \\ Science Applications, Inc. \\ 5 Palo Alto Square, Suite 200 \\ Palo Alto CA 94304
}

\begin{abstract}
A white dwarf model with $M=.6 \mathrm{M}_{\odot}, \mathrm{T}_{\mathrm{e}}=12000 \mathrm{~K}$, and $\mathrm{L}=1.2 \times 10^{31}$ erg $\sec ^{-1}$ provided by A.N. Cox has been tested for linear stability of radial oscillations. The radial mode instability first reported for this model by Cox, et. al (1979) has been confirmed. The growth rates obtained are comparable to the rates found by Cox. A sequence of $l=2 \mathrm{~g}-$ modes has also been found to be unstable. The e-folding times range from around $10^{11}$ periods for a 137 second mode ( 1 radial node) to less than 100 periods for a 629 second mode ( 17 nodes). It is 1ikely that the latter rate is too high because the elgenfunction has been forced to vanish at the non-zero inner radius of the model, at which the BruntVäisäla frequency is barely less than the mode frequency.
\end{abstract}

\section{THE MODEL}

The model used in this study was constructed by Cox, et. al (1979) in a study of $\mathrm{ZZ}$ Ceti varlables. The composition layers from the surface inward are: $\mathrm{Y}=0.28, \mathrm{Z}=0.02$ to $\mathrm{T}=10^{6} \mathrm{~K} ; \mathrm{Y}=0.58, \mathrm{Z}=0.02$ to $2 \times 10^{6} \mathrm{~K}$; $Y=0.98, Z=0.02$ to $5 \times 10^{6} \mathrm{~K} ; \mathrm{Y}=0.90, \mathrm{Z}=0.1$ (carbon) to $10^{7} \mathrm{~K} ; \mathrm{Y}=0.50$ $\mathrm{z}=0.5$ (carbon) to $\rho=10^{4} \mathrm{gm} \mathrm{cm}^{-3}$; the remainder is pure carbon. The model has an outer radius of $9 \times 10^{8} \mathrm{~cm}$, and is cut off at an inner radius of $2.4 \times 10^{8} \mathrm{~cm}$. The mass of the omitted core $1 \mathrm{~s} 10^{32} \mathrm{gm}$. The internal luminosity varies in proportion to the mass. A convection zone occurs between $\mathrm{T} \simeq 10^{4} \mathrm{~K}$ and $2.7 \times 10^{4} \mathrm{~K}$; however, it carries 1 ess than $0.5 \%$ of the flux at most. For purposes of the stability calculation the entire flux in this region has been attributed to radiation.

The model structure including opacity derfvatives and thermodynamic quantities required for the stability analysis were specified on a grid of 195 points chosen by Cox.

\section{CALCULATIONS}

The eigenfunction and stability calculations were done on several different grids, obtained by interpolation (spline and linear) from the initial model. The abrupt changes in composition were somewhat washed out by this procedure: no effort was made to represent them sharply. 
In particular, the squared Brunt-Väisäla frequency is formally $\pm \infty$ at discontinuities in density; however, it was interpolated smoothly across such points. The expression actually used was

$$
\omega_{B V}^{2}=\frac{g^{2} \Gamma_{l}}{c^{2}}\left(\nabla_{A}-\nabla\right)
$$

in which

$$
Q \equiv-\left.\frac{\partial \ln }{\partial \ln } \frac{\rho}{T}\right|_{P}
$$

and the other symbols have their usual meanings. Eq. (1) is valid in regions of fixed composition, as is true in a plecewise sense in the present model. The Eulerian perturbation of the gravitational potential was neglected.

The adiabatic elgenfunctions were calculated by the inverse iteration method, and were used to calculate the Lagrangian perturbation of the divergence of the heat flux. The Lagrangian perturbation was calculated as directly as possible to avoid the considerable cancellation which sometimes occurs between the Eulerian perturbation and the $\vec{v} \cdot \nabla$ term for quantities which vary rapidly in space in the initial model. A brief discussion of the actual equations used was given by Keeley (1979), so no details are repeated here.

The displacement eigenfunction and $\delta(\nabla \cdot \vec{F})$ were set to zero at the inner boundary, for the calculations described below. At the outer boundary, the displacement vector was elther matched to an outgoing evanescent wave, or the Lagranglan pressure perturbation was set to zero. The exterior boundary condition on the flux perturbation was $(4 \pi K \rho)^{-1}$ $\delta(\nabla \cdot \vec{F})=\delta B$, in which the perturbations are Lagranglan, $K$ is the opacity, and $B$ is the Planck function. This condition was discussed by Keeley (1977).

\section{RESULTS}

\section{a. Radial Modes}

Since these calculations serve mainly to confirm the results presented earlier by Cox, et. al (1979), they are not discussed in detall.

Eigenfunctions with zero to twelve nodes were calculated on several 200 point grids. The lowest few nodes ( periods $\geqslant 3$ seconds) were stable, with damping times $\sim 10^{14}$ perlods. Instability with e-folding times on the order of $2 \times 10^{9}$ periods was found for high modes $(\mathrm{e.g} ., 10$ radial nodes), in reasonable agreement with Cox, et. al.

Driving regions were located near roughly $1.7 \times 10^{5} \mathrm{~K}$, between about $4 \times 10^{4}-7 \times 10^{4}$, and between about $1.6 \times 10^{4}-3 \times 10^{4}$. The latter two together provided the necessary excitation; in general neither one alone was sufficlent to overcome the damping. Although convective flux was ignored, the damping by turbulent viscosity was estimated as described by Goldrelch and Keeley (1977). In no case was it important. The evanescent wave boundary condition produced slightly stronger driving. 
Some p-modes with spherical harmonic index $l=2,10,50$, and 100 were also checked. The $l=2$ mode with three radlal nodes was stable with a damping time of $\sim 10^{7}$ years $\left(\sim 10^{14}\right.$ periods $)$ but the 4 and 5 node oscillations were unstable. At $2=10$, the switch from stability to instabllity occurred between 2 and 3 radial nodes. At $l=100$, the lowest mode was just barely unstable. Thus 1 t appears that the general pattern found for the radial nodes 18 repeated for the $p$-modes.

\section{c. g-Modes $(l=2)$}

The full sequence of modes between 137 seconds and 629 seconds (17 radial nodes) was found to be unstable. Growth times decreased systematically from $\sim 10^{10}$ periods to $\sim 100$ pertods (at 629 seconds). The Brunt-Välsäla frequency at the inner boundary of the model is $7 \times 10^{-3}$ $\mathrm{sec}^{-1}$, and rises quickly above $1 \times 10^{-2}$, the angular frequency corresponding to 629 seconds. Thus the interior boundary condition suppressed the elgenfunction artificlally in the deep interior. The damping in the Inner half of the radius of the shell comprising the model was many orders of magnitude less than that just below the main driving regions. Even an order of magnitude increase in amplitude near the inner boundary would not likely influence the net excitation (ergs/sec.) very much. However, the kinetic energy associated with a larger interior motion could easily be a dominant fraction of the total (for fixed surface amplitude), and this would result in a longer e-folding time without any tendency to change the sign from excitation to damping.

The driving reglons are essentially the same as those identified for the radial modes by Cox, et. al (1979). It is impossible to make a clean separation of radial and non-radial effects in the driving or damping. Both radial and horizontal flux perturbations depend on the divergence of the displacement vector, which in turn depends on both radial and horizontal motions. In the 415 second (10 node) mode, both motions contributed substantially to the divergence of the displacement. On the other hand, the contribution of the horizontal Lagranglan flux perturbation to the divergence of the total flux was typically at the $1 \%$ level. The contribution to the driving due to the positive flux divergence in the initial model was negligible.

The outgoing evanescent wave condition was used in all cases. For the 415 second mode, the alternative boundary condition resulted in a $7 \%$ decrease in the growth rate. Since none of the mode frequencles considered here are very close to the critical frequencles at the surface, it is unlikely that much larger effects than this will occur for the other modes.

Al1 the modes of the sequence were calculated on 200 point grids, and several were calculated with 350 points. The difference in growth rates between a good 200 point grid and the 350 point grid with the same relative distribution of points was generally less than $5 \%$. 
d. g-Modes for Higher $\ell$

A few g-modes were calculated for $\ell=3,4,10$, and 50 , with periods in the range 10-200 seconds. All were found to be unstable, with growth rates roughly comparable to $l=2$ modes with the same number of radial nodes.

\section{CONCLUDING REMARRS}

Many careful calculations in the past have failed to find g-mode instabilities, although the observational evidence for their existence is extensive [see Van Horn (1979) for a brief discusssion and references]. The success of the present calculations must be attributed to the approprlateness of the model constructed by Cox, et. al. However, if so many modes are linearly unstable as has been found here, it w111 be necessary to proceed to non-linear calculations in order to understand the fact that only one or a few modes are generally seen to be excited.

I thank A.N. Cox and his collaborators for supplying the model used in this investigation. These calculations were done with a computer program developed with the support of NASA grant NSG7342 for studying solar oscillations.

\section{REFERENCES}

Cox, A.N., Hodson, S.W., and Starrfield, S.G., 1979, Proceedings of the Non-radial and Non-linear Stellar Pulsation Workshop, Tucson, Arizona, 12-16 March.

Goldre1ch, P., and Keeley, D., 1977, Ap. J. 211, 934.

Keeley, D., 1977, Proceedings of the Symposium on Large Scale Motions on the Sun, Sacramento Peak Observatory, Sunspot, New Mexico, 1-2 Sept.

Keeley, D., 1979, Proceedings of the Non-radial and Non-Linear Stellar Pulsation Workshop, University of Arizona, Tucson, Arizona, March 12-16.

Van Horn, H.M., 1979, Physics Today, Volume 32, \#1, p. 23, January. 